\title{
Unforced polymer translocation compared to the forced case
}

\author{
V. V. Lehtola, R. P. Linna, and K. Kaski \\ Department of Biomedical Engineering and Computational Science, Helsinki University of Technology, P.O. Box 9203, \\ Helsinki FIN-02015 TKK, Finland
}

(Received 22 December 2009; published 23 March 2010)

\begin{abstract}
We present results for unforced polymer translocation from simulations using Langevin dynamics in two dimensions (2D) to four dimensions and stochastic rotation dynamics supporting hydrodynamic modes in three dimensions (3D). We compare our results to forced translocation and a simplified model where the polymer escapes from an infinite pore. The simple model shows that the scaling behavior of unforced translocation is independent of the dimension of the side to which the polymer is translocating. We find that, unlike its forced counterpart, unforced translocation dynamics is insensitive to pore design. Hydrodynamics is seen to markedly speed up the unforced translocation process but not to affect the scaling relations. Average mean-squared displacement shows scaling with average transition time in unforced but not in forced translocation. The waiting-time distribution in unforced translocation follows closely Poissonian distribution. Our measured transfer probabilities align well with those obtained from an equilibrium theory in $3 \mathrm{D}$, but somewhat worse in $2 \mathrm{D}$, where a polymer's relaxation toward equilibrium with respect to its translocation time is slower. Consequently, in stark contrast to forced translocation, unforced translocation is seen to remain close to equilibrium and shows clear universality.
\end{abstract}

DOI: 10.1103/PhysRevE.81.031803

PACS number(s): 82.35.Lr, 87.15.-v, 87.15.A-

\section{INTRODUCTION}

The transport of biopolymers through membranes is present in many biological systems [1]. Motivated by its potential applicability in ultrafast DNA and RNA sequencing [2-5], and protein import into mitochondrion [6], most of the experimental research has been focused on forced polymer translocation. However, biological translocation processes where a strong driving field is not present have motivated studies on unforced translocation [7]. Partly due to the abundance of detailed processes adding to the complexity of its dynamics, such as binding of specific proteins on either the cis or trans side of the membrane, and the effects due to the properties of the pore and the solvent, experimental studies on unforced translocation are few [8] compared with the number of computational studies (see, e.g., [9-16]). In spite of the extensive research, even the dynamics of the generic process still remains relatively poorly understood. Hence, the characterization of the process in connection with simpler or better understood models seems to be called for.

In unforced translocation, the average polymer translocation (or escape) time $\tau$ has been observed to scale with respect to the polymer length $N$ as $\tau \sim N^{\beta}$. For the scaling exponent $\beta$ Langevin dynamics (LD) and Monte Carlo simulations give the identical value of 2.5 in two dimensions (2D) $[9-11,17]$. This value is in accordance with the predicted lower limit $\beta=2 \nu+1$ based on the free diffusion of the center of mass of the polymer [9]. Here, the swelling exponent $\nu$ has the value of 0.75 for a polymer in $2 \mathrm{D}$. Recently it has been argued that this lower limit should be the actual value of the scaling exponent for unforced translocation [17]. Accordingly, in three dimensions (3D), where $\nu=0.588$, this would yield $\beta \simeq 2.18$. Results from Langevin dynamics simulations, $\beta=2.23 \pm 0.03$ [18] and $\beta=2.22 \pm 0.06$ [17], and dynamic Monte Carlo simulations [19], $\beta=2.23 \pm 0.04$, support this argument also in 3D. However, there exist con- tradicting results. In $3 \mathrm{D}, \beta=2.5$ was obtained $[12,13]$, for which two independent explanations were proposed. Anomalous diffusion was dealt with by fractional Fokker-Plank equation framework in [12], whereas a simple scaling proposition $\beta=2+\nu$ based on excess monomer density near the pore was suggested in [13]. In addition, $\beta=2.27 \pm 0.04$ was obtained from a three-dimensional coarse-grained moleculardynamics simulation where hydrodynamics (HD) was included [14]. In 3D free diffusion was claimed to govern the process. However, since the prediction based on free diffusion and using the Zimm model (as hydrodynamics is included) gives $\beta=3 \nu \simeq 1.77$, the result is in agreement only with the original lower-limit argument [9]. This lower limit is then the only theoretical result with which all the presented computational results agree.

Here, we study unforced translocation using both stochastic rotation dynamics (SRD) and LD. This study is motivated by our previous findings on forced translocation [20,21] upon which we will reflect our present results. We provided evidence for the forced translocation as being a highly nonequilibrium process and explained the observed dynamics by a simple force balance, valid in the experimentally and biologically relevant force range. Based on purely analytical calculations, arguments along similar lines have previously been given by Sakaue [22].

Longer polymers are more prone to translocate fast enough that the translocated segments will not have time to relax to equilibrium [9]. In view of this and our previous observations on forced translocation, it is important to evaluate the equilibrium assumption of unforced polymer translocation. Since hydrodynamics drastically changes the scaling of average translocation time with respect to the chain length and reduces the translocation times in the forced translocation, first reported in [23] and then by us [21], it is also of importance to evaluate its significance for unforced translocation. A previous study reports a negligible effect of hydrodynamics on unforced translocation [14], whereas somewhat 


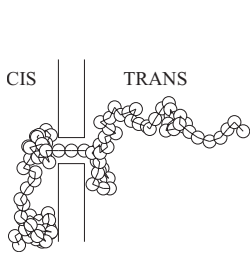

(a)

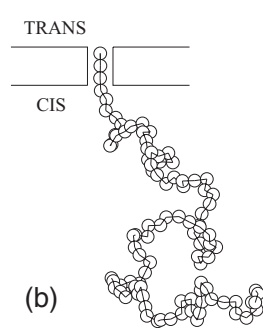

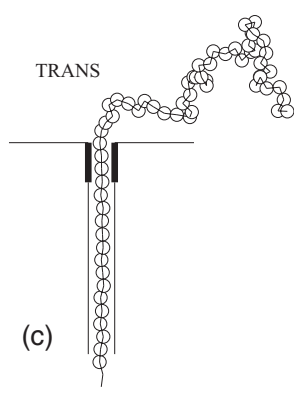

FIG. 1. Snapshots of (relaxed) initial configurations in 3D for (a) unbiased translocation with $N=101$ (so initially $s=51$ ), (b) forced translocation with $N=100$, and (c) unforced pore escape with $N=101$ (not all of the beads inside the quasi-one-dimensional tunnel are shown). Distance $3 b$ from the pore opening is marked with bold lines.

larger effect was seen in [15]. In the present study we evaluate the effect of hydrodynamics on the unforced translocation when hydrodynamic modes on the trans and cis sides are decoupled [see Fig. 1(a)].

In addition to forced translocation we shall also reflect the unforced translocation upon the so-called "pore escape" problem, a special case of unforced translocation, where the entropic effects are eliminated from one side of the wall. Theoretical interest of this model comes from it relating to the biologically relevant Brownian ratchet mechanism [7]. It is also of interest for understanding the intracellular transport processes through microtubules.

The present paper is organized as follows. In Sec. II the equilibrium translocation theory is briefly reviewed. Section III describes the polymer model used in our simulations. In Sec. IV the implemented translocation models are described. In Sec. $\mathrm{V}$ results are reported and discussed, and summary and conclusions are made in Sec. VI.

\section{EQUILIBRIUM THEORY}

We will make comparison to the equilibrium framework describing the polymer translocation introduced by Sung and Park [24], and Muthukumar [25]. In this framework the translocating polymer segments on the cis and trans sides are treated as two thermodynamic ensembles separated by a wall. The polymer translocates from the cis to the trans side through a narrow pore in the wall (see Fig. 1). The partition sum $Z_{n}$ of a polymer with $n$ monomers in a semi-infinite space bounded by a hard wall to which one end of the polymer is attached is given by [26]

$$
Z_{n} \sim \mu^{n} n^{(\gamma-1)}
$$

where $\gamma=0.69$ for the attached self-avoiding chain and $\mu$ is the connective constant. Assuming that this polymer segment consisting of $n$ monomers is in thermal equilibrium, its free energy can be written as $F_{n}=-k_{B} T \ln Z_{n}$. Now, if the segments of the translocating polymer, $s$ and $N-s$ monomers long, on either side of the wall are in separate thermal equilibria, the free energy for the whole polymer can be written as

$$
\frac{F_{s}}{k_{B} T}=(1-\gamma) \ln [s(N-s)] .
$$

Here, the pore is assumed to be short, so the beads inside it need not be taken into account.

\section{POLYMER MODEL}

The standard bead-spring chain is used as a coarsegrained polymer model with both LD and SRD methods. In this model adjacent monomers are connected with anharmonic springs, described by the finitely extensible nonlinear elastic potential,

$$
U_{F E N E}=-\frac{K}{2} R^{2} \ln \left(1-\frac{r^{2}}{R^{2}}\right),
$$

where $r$ is the length of an effective bond and $R=1.5$ is the maximum bond length. The Lennard-Jones (LJ) potential,

$$
\begin{gathered}
U_{L J}=4 \epsilon\left[\left(\frac{\sigma}{r}\right)^{12}-\left(\frac{\sigma}{r}\right)^{6}\right], \quad r \leq 2^{-1 / 6} \sigma, \\
U_{L J}=0, \quad r>2^{-1 / 6} \sigma,
\end{gathered}
$$

is used between all beads of distance $r$ apart. The parameter values were chosen as $\epsilon=1.2, \sigma=1.0$, and $K=60 / \sigma^{2}$. The used LJ potential with no attractive part mimics good solvent.

\section{TRANSLOCATION MODELS}

Langevin dynamics model. We use our translocation model based on Langevin dynamics [20] to perform simulations of unforced translocation in dimensions $d \in[2,4]$. Hence, the time derivative of the momentum of bead $i$ reads as

$$
\dot{\mathbf{p}}_{i}(t)=-\xi \mathbf{p}_{i}(t)+\eta_{i}(t)+f\left(\mathbf{r}_{i}\right),
$$

where $\xi, \mathbf{p}_{i}(t), \eta_{i}(t)$, and $f\left(\mathbf{r}_{i}\right)$ are the friction constant, the momentum, random force of the bead $i$, and the external force (constant, but exerted only inside the pore), respectively. Naturally, $f=0$ for unforced translocation. $\xi$ and $\eta_{i}(t)$ are related by the fluctuation-dissipation theorem.

The dynamics was implemented using the velocity Verlet algorithm [27]. The wall containing the pore is a continuous infinite potential barrier. The pore, aligned with the $z$ axis, is of diameter $a$ and length $3 \times a$, where $a=1$ is the Kuhn length of the modeled polymer. It was implemented using a damped harmonic potential that centers the beads toward the $z$ axis. In this pore design the dimension $d \geq 2$ can be easily changed. In our simulations $\xi=0.73$ and the polymer bead mass $m=16$, and $k_{B} T=1$, where $k_{B}$ is the Boltzmann constant and $T$ is the temperature. Then, at long times according to Einstein's relation, the one-particle self-diffusion constant is given by $D_{0}=k_{B} T / \xi m \approx 0.086$. Time steps of 0.01 and 0.03 were used in the forced and unforced simulations, respectively.

Stochastic-rotation-dynamics model. Simulations including hydrodynamics were performed in 3D using SRD [28]. 
Our SRD translocation model has been previously used for simulating forced translocation [21]. Except for the wall and the pore regions, the simulation space is filled with fictitious solvent particles. The dynamics consists of consequent collision and streaming steps. The solvent space is divided into cells, within which the fictitious solvent particles perform simplified dynamics where collisions among them and with the polymer beads are taken effectively into account by randomly rotating the noncollective (random) parts of their velocities. Hence, the collision step can be written as

$$
\mathbf{v}_{i}\left(t+\Delta t_{S R D}\right)=\underline{\mathbf{R}}\left[\mathbf{v}_{i}(t)-\mathbf{v}_{c m}(t)\right]+\mathbf{v}_{c m}(t),
$$

where $\mathbf{v}_{i}$ are the particle velocities inside a cell, $\Delta t_{S R D}$ is the time step for solvent dynamics, $\underline{\mathbf{R}}$ is the rotation matrix, and $\mathbf{v}_{c m}$ is the center-of-mass velocity of the particles within the cell. Hydrodynamic modes are supported over the cells resulting in a finite Reynolds number. The collision step is followed by the free-streaming step where particle $i$ 's position $\mathbf{r}_{i}$ is moved according to

$$
\mathbf{r}_{i}\left(t+\Delta t_{S R D}\right)=\mathbf{r}_{i}(t)+\mathbf{v}_{i}(t) \Delta t_{S R D} .
$$

Thermostating is done by rescaling all solvent particle velocities, so that equipartition theorem holds at all times. The polymer beads move according to detailed molecular dynamics. In addition, they take part in the above-described SRD steps that are performed every 50th molecular-dynamics time step.

A slit of width $25 a$ for $N \leq 31$ or $32 a$ for $N>31$ is formed by two aligned plates in the $y z$ plane. The solvent-filled space between the plates is divided by a wall in the $x y$ plane. This wall contains the pore. The simulation space is $32 a$ wide in $y$ and $z$ directions. These directions are periodically connected. All walls are continuous and impose a no-slip boundary condition to the fluid particles. Inside the pore polymer beads perform only molecular dynamics. The diameter of the cylinder-shaped pore is $1.2 a$. Exactly as in the LD based model, the pore contains a damped harmonic potential centering the beads toward the axis of the cylinder. Also in this model $a=1$ and $k_{B} T=1$.

In both models the polymer having an odd number of beads $N$ is initially placed halfway inside the pore [see Fig. 1(a)]. The polymer is then let relax for a time longer than its Rouse relaxation time. We register events when the segment $s=s_{0}$ is replaced with segment $s_{0}-1$ or $s_{0}+1$ in the middle of the pore. The polymer is considered translocated, when it has completely exited the pore to the trans side.

\section{RESULTS}

\section{A. Scaling with polymer length}

As our implementation of the pore and the continuous wall differs from the most frequently used type of implementation based on immobile "particles," we first check if it has effect on the scaling relation for unforced translocation, $\tau$ $\sim N^{\beta}$, where $\tau$ is the translocation time or the time it takes for the polymer to exit the pore, $N$ is the polymer length, and $\beta$ is the scaling exponent. This is crucial for validating our previous results for forced translocation $[20,21]$ as there ex-
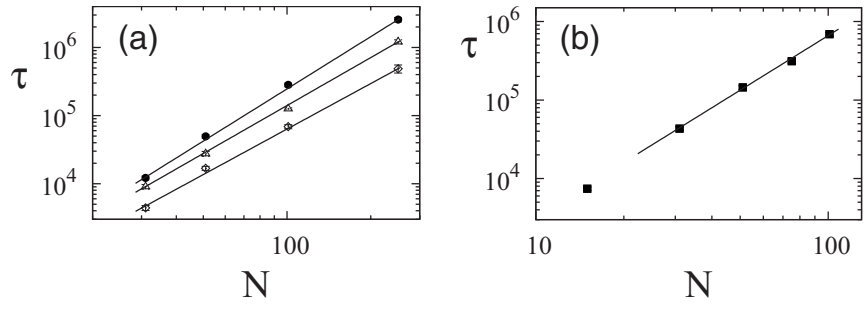

FIG. 2. Scaling of the translocation time $\tau$ with respect to the chain length $N$ for unforced translocation. (a) Results without hydrodynamics from Langevin dynamics simulations. The scalings $\tau$ $\sim N^{\beta}, \beta=2.55 \pm 0.05,2.33 \pm 0.05$, and $2.26 \pm 0.05$ are obtained in $2 \mathrm{D}, 3 \mathrm{D}$, and $4 \mathrm{D}$, respectively (from top to bottom). The fourdimensional curve has been shifted down for clarity. For a fixed length $N$ the translocation actually becomes faster as $d$ increases. (b) Here, using SRD with hydrodynamics we obtain the scaling exponent $\beta=2.30 \pm 0.07$.

ists at least a reasonable agreement on the scaling exponents in the unforced case.

For unforced translocation in 2D, we obtain $\beta$ $=2.55 \pm 0.05$, shown in Fig. $2(\mathrm{a})$, which is in good agreement with the scaling result of $\beta=2.5$ obtained by others [9-11]. In $3 \mathrm{D}$, we have $\beta=2.33 \pm 0.05$ from LD simulations [see Fig. 2(a)], which falls between the results $\beta \simeq 2.23$ in [17-19] and $\beta=2.5$ in $[12,13]$. Hence, our model produces the to some extent established scaling relations for the unforced translocation, which may potentially be universal. Hence, our previous arguments for the strong nonequilibrium nature of the forced translocation remain valid.

Making use of our pore implementation in the LD translocation model, we obtain $\beta=2.26 \pm 0.05$ in the dimension of 4 [see Fig. 2(a)]. Of the previously introduced scaling arguments, $\beta=2 \nu+1$ [9] and $\beta=2+\nu$ [13], only the first remains as a valid lower limit in four dimensions (4D), where $\nu$ $=0.5$ (ideal chain).

In addition to the scaling, the data in Fig. 2(a) show that the translocation of a chain of fixed length becomes faster as the number of dimensions increases from two to four. For example, for fixed $N=31, \tau=12161 \pm 768$ (2D), $9020 \pm 721$ (3D), and $6613 \pm 537$ (4D). This follows from the equipartition theorem and the increase in the diffusion coefficient $D$ with increasing dimension $d$. (For one-particle diffusion the diffusion constant depends linearly on dimension, $D \sim d$.)

In order to characterize the effect of hydrodynamics on unforced translocation in 3D, we use our SRD model, which allows for switching hydrodynamics on and off. The translocation time $\tau$ was computed both with and without hydrodynamics, keeping $N=31$ fixed. With HD the translocation time of the polymer is clearly smaller, $\tau=42000 \pm 3000$, than without HD, $\tau=99000 \pm 8000$. However, hydrodynamics does not have a strong effect on the scaling of the translocation time, $\tau \sim N^{\beta}$. We obtain $\beta=2.30 \pm 0.07$ with HD [see Fig. 2(b) $]$ and $\beta=2.33 \pm 0.05$ without HD. A slightly lower value for the scaling exponent $\beta$ with hydrodynamics is logical since the long-range correlations should diminish the effect of chain length to translocation dynamics. Hence, the effect of hydrodynamics is much weaker for unforced than forced translocation [20,21] in accord with the argued highly nonequilibrium nature of the latter. 

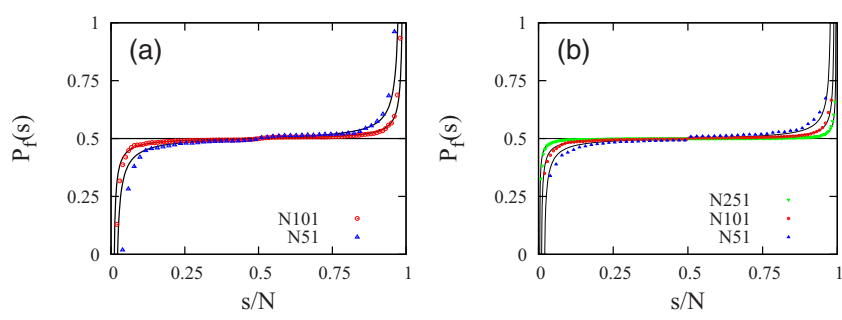

FIG. 3. (Color online) The forward move (cis $\rightarrow$ trans) probability $P_{f}(s)$ for chains of length $N=51$ and 101 for unforced translocation. The solid lines are theoretical predictions from Eq. (8), derived using the equilibrium free energy. The reduction in $N$ due finite pore length has been accounted for by using values of $N$ $\in\{49,99\}$ in theoretical predictions. (a) SRD results where hydrodynamics is included and the pore is frictionless. The best fit (shown) is obtained with $\gamma=0.69 \pm 0.05$. (b) Results from Langevin dynamics in 2D with frictional pore, $N=51,101$, and 251 (from top to bottom at the right side of the figure). Hence, the probability curves asymptotically approach the flat $P_{f}=0.5$ line as $N$ is increased. The best fit (shown) is obtained with $\gamma=0.80 \pm 0.05$.

Our scaling exponent $\beta=2.30 \pm 0.07$ with HD agrees with $\beta=2.27 \pm 0.04$ for $N \in[3,31]$ obtained in [14]. In contrast to the model used there, in our model the solvent does not enter the pore. Hence, in our model the long-range hydrodynamic modes are not supported across the pore and, accordingly, whether the solvent enters the pore or not does not seem to affect the scaling of $\tau$ with $N$.

\section{B. Transfer probabilities}

Next, we investigate transition probabilities in the unforced translocation process. The polymer beads are numbered from 1 to $N$, with the middle bead initially in the pore and the end bead 1 on the trans and $N$ on the cis side. The states of the system are labeled by the reaction coordinate $s$, defined as the number of the polymer bead currently in the middle of the pore. The system enters the state $s$, when the bead number $s$ enters the middle of the pore. Assuming that the two polymer segments on each side of the wall are in separate thermal equilibria, we can calculate the transfer probability of a "forward move" as $P_{f}(s)=P(s \rightarrow s+1)$ $\sim \exp \left[-\beta\left(F_{s+1}-F_{s}\right)\right]$, where $\beta=1 / k_{B} T$ and $F_{s}$ is the free energy given by Eq. (2). We obtain

$$
P_{f}(s) \propto\left(1-\frac{1}{s}+\frac{1}{N-s}\right)^{1-\gamma} .
$$

In our simulations, the pore is $3 a$ long, so beads $s-1$ and $s$ +1 are inside the pore, which is taken into account by using effective values $s-1$ and $N-s-1$ for the chain lengths on trans and cis sides in Eq. (8).

In Fig. 3(a) the transfer probabilities obtained from the SRD simulations in 3D are compared to those given by Eq. (8). The best fit of the probabilities from both LD and SRD simulations to the analytical values is obtained for the exponent value $\gamma=0.69 \pm 0.05$, which is the exponent for the selfavoiding walk (SAW) and hence in agreement with our measured value for the swelling exponent, $\nu=0.60 \pm 0.02$, which for $\mathrm{SAW}$ is $\nu=0.588$. Consequently, in $3 \mathrm{D}$ the unforced translocation is adequately described by two thermodynamic ensembles separately in thermal equilibrium, even in the presence of hydrodynamic modes. However, close to the chain ends, i.e., when $s$ is close to 1 or $N$, translocating segments are inclined to accelerate due to the large entropic difference of the polymer segments on the two sides of the wall resulting in a large driving force. This has been studied in detail elsewhere [16].

Unlike in 3D, in two dimensions the transfer probabilities obtained from LD simulations cannot be fitted to those expected from the equilibrium theory. The best-fit exponent value $\gamma=0.80 \pm 0.05$ deviates substantially from the analytically obtained exact value $\gamma=61 / 64 \approx 0.95$ [29]. Since the connective constant $\mu=\lim _{n \rightarrow \infty} \mu_{n}$ in Eq. (1) is defined only in the limit $n \rightarrow \infty$ and could otherwise present a nontrivial dependence on the reaction coordinate $s$ in Eq. (8), we calculated the values for $\mu_{n}$ for small $n$ from SAW enumeration data provided by the authors of [30] on their webpage [31]. However, in a half space, relevant for our simulation space divided by the wall, the value of $\mu_{n}$ saturates to a constant at very short walks $n$, and hence the finite polymer length $N$ $=n$ does not have a significant contribution via $Z_{n}$ on $P_{f}(s)$.

Since the potential contribution from finite-size modification of equilibrium configuration turned out to be weak, we are left with the possibility that the deviation of $\gamma$ obtained by fitting the measured $P_{f}$ to Eq. (8) might be due to the unforced translocation in 2D taking place out of equilibrium. We measured the relaxation times of grafted polymers of lengths $N \in[30,100]$ in $2 \mathrm{D}$, where polymer's relaxation toward equilibrium is slower than in $3 \mathrm{D}$. The measured autocorrelation shows exponential relaxation in both dimensions, $\bar{G}\left(r, t-t^{\prime}\right) \sim \exp \left[-\left(t-t^{\prime}\right) / \tau\right]$, where the overbar denotes average over all polymer beads. The relaxation times in $2 \mathrm{D}$ and $3 \mathrm{D}$ are related as $\tau_{2 d}^{R} / \tau_{3 d}^{R} \approx 1.7$ and $\approx 2.4$ for $N=30$ and $N$ $=100$, respectively. The translocation times are related as $\tau_{2 d} / \tau_{3 d} \approx 1.6, \approx 2.0$, and $\approx 2.4$ for $N=31,101$, and 251 , respectively. The relative increases in $\tau$ and $\tau^{R}$ with respect to $N$ imply that long polymer chains are more prone to remain out of equilibrium during the translocation in $2 \mathrm{D}$ than in $3 \mathrm{D}$. This would offer an explanation to the deviation of the measured exponent $\gamma$ from the exact value obtained from the equilibrium framework.

\section{Average transition time distribution}

Next, we determine the distribution of the average transition time $\langle t\rangle$ for the system to move from one state to another, i.e., $s \rightarrow s-1$ or $s \rightarrow s+1$. Taking the average over 100 simulation runs we obtain the distributions of the transition time $\pi(\langle t\rangle)$ for each chain length $N$ (see Fig. 4). The distributions are normalized such that they cover a unit area. The distributions both fit Poissonian-like probability density functions of the form $P(\widetilde{t})=A t^{\lambda} \exp (-k \widetilde{t})$, where $\tilde{t}=t-t_{0}$ and $t_{0}$ is a fitting parameter that determines the minimum time for a transition to occur. The use of the minimum time parameter $t_{0}$ is justified by the beads being connected by springs of finite length which must be traversed prior to any transition.

The distribution of average transition times $\langle t\rangle$, i.e., waiting-time distribution, does not converge to a Poissonian 

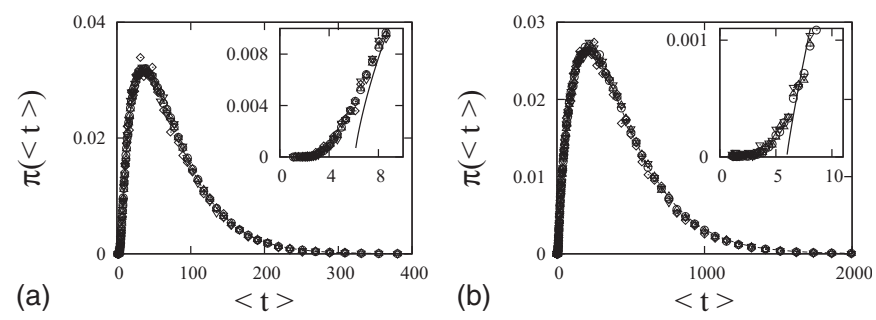

FIG. 4. The distribution of average state transition times $\langle t\rangle$ for unforced translocation. Distributions are from (a) three-dimensional Langevin dynamics with $N=31,51,101,251$ (distributions in 2D and 4D are similar) and (b) SRD simulations with hydrodynamics with $N=31,51,75,101$. All the distributions are almost Poissonian, except for the deviation at small values of $\langle t\rangle$; see the magnifications in the insets. The time units in (a) and (b) are model dependent and cannot be straightforwardly compared. See text for details.

distribution in detail, as can be seen from the insets of Figs. 4(a) and 4(b). The correlations due to adjacent polymer beads being connected are enhanced at high translocation velocity, i.e., small $\langle t\rangle$, deviating the distribution $\pi(\langle t\rangle)$ from Poissonian there. Using SRD, similar characteristics are obtained with and without hydrodynamics in 3D and, using LD, without hydrodynamics in dimensions $2 \leq d \leq 4$. Apart from the deviation at small $\langle t\rangle$, the distributions are Poissonian. The distribution's exponential tail $\exp (-k \widetilde{t})$ falls off faster as $d$ is increased. To determine the effect of hydrodynamics on the distribution, we compare the results between the 3D LD and SRD models by using a temporal reference value $\tau$ for $N=31$ without hydrodynamics in order to make the values of $k$ from Fig. 4 dimensionless, $k^{*}=k \tau_{N 31}$. We get $k_{S R D}^{*} \simeq 442$ $\left(\tau_{S R D, N 31}=99000 \pm 8000\right) \quad$ and $\quad k_{L D}^{*} \simeq 230 \quad\left(\tau_{L D, N 31}\right.$ $=9020 \pm 720)$, implying that the tail of the average transition time distribution $\pi(\langle t\rangle)$ decays faster with hydrodynamics. In other words, hydrodynamics decreases fluctuations of transition times through increased range of correlation.

\section{Mean-squared displacement}

For the scaling of the mean-squared displacement of the reaction coordinate $s,\left\langle\delta s^{2}(t)\right\rangle \sim t^{\alpha}$, in Fig. 5(a), we obtain $\alpha=0.81 \pm 0.02$ and $\alpha=0.88 \pm 0.03$ in dimensions of 2 and 3 , respectively. These results agree well with the theoretical prediction, $\alpha=2 /(2 \nu+1)$, by Chuang et al. [9], from which $\alpha$ is 0.8 in $2 \mathrm{D}, 0.92$ in $3 \mathrm{D}$, and their previous computational confirmation with LD [17], $\alpha=0.80 \pm 0.01$ (2D) and $0.91 \pm 0.01$ (3D). Additionally, we obtain $\alpha=0.90 \pm 0.05$ in dimension of 4 (not shown). Since $d_{c}=4$ is the upper critical dimension for a self-avoiding random walk [32], $\alpha$ is not expected to change when increasing the dimension beyond this. We checked that the swelling exponent $\nu$ for both a free and a grafted polymer decreases as a function of the dimension in our model. For chains of length $N \in[25,200]$ we obtained swelling exponents $\nu=0.75 \pm 0.02,0.60 \pm 0.02$, and $0.53 \pm 0.02$ in dimensions of 2,3 , and 4 , respectively, in agreement with those for self-avoiding random walks, $\nu=0.75,0.588$ in $d=2,3$, respectively, and for an ideal chain, $\nu=0.50$, in $d \geq 4$ [33].
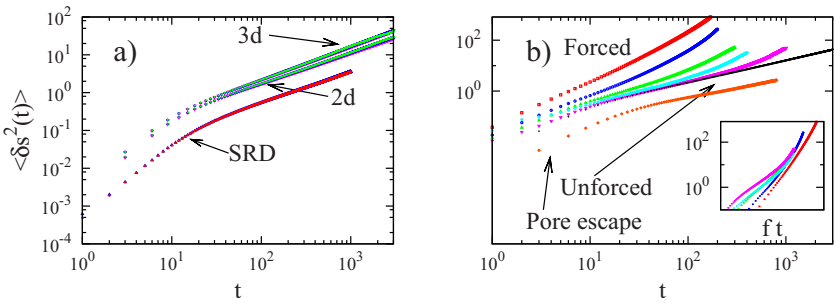

FIG. 5. (Color online) The mean-squared displacement of the reaction coordinate $s$. (a) Unforced translocation. We obtain slopes of $0.81 \pm 0.02$ in $2 \mathrm{D}$ and $0.88 \pm 0.03$ in $3 \mathrm{D}$ for Langevin simulations, $N \in\{51,101,251\}$, and $0.83 \pm 0.01$ for 3D SRD simulations including hydrodynamics, $N \in\{51,101\}$. Collapsed data when $\left\langle\delta s^{2}(t)\right\rangle \sim t^{\alpha}$ is normalized by $N$. (b) In $3 \mathrm{D}$, data from LD simulations for various cases; forced and unforced translocations and pore escape. For forced translocation, $f=10,3,1,0.7$, and 0.3 , respectively, starting from the top. The slope is about 0.92 for $f=0.3$ and is increased up to 2.2 for $f=10$. The mono-black-plot, second from the bottom, represents unforced translocation data. The diamonds at bottom represent the pore escape data. Here, $N=200$ for forced cases and 251, otherwise. Inset: the data for forced translocation does not collapse with respect to the force $f$, nor with respect to the chain length $N$ (not shown).

The effect of hydrodynamics, which showed relatively weak on the scaling of translocation time with polymer length, is more pronounced on the variance of the reaction coordinate. At long times a power law is obtained, $\left\langle\delta s^{2}(t)\right\rangle$ $\sim t^{\alpha}$, with $\alpha=0.83 \pm 0.02$ and $\alpha=0.88 \pm 0.03$ with and without hydrodynamics, respectively [see Fig. 5(a)]. This is in agreement with long-ranged hydrodynamic correlations, diminishing transition time fluctuations over larger length and time scales. Hence, hydrodynamics has no effect on transition probabilities $P_{f}$, as noted in the previous section, but changes only slightly the time distribution of state transitions. This is in contrast to the forced translocation where hydrodynamic correlations mediate the applied pore force along the chain, thus enhancing the collective motion of the chain and so having influence not only on the variance but also the average of the translocation time.

\section{E. Comparison to pore escape and forced translocation}

In order to pin down the difference between the underlying dynamics of the unforced and forced translocation processes, we present the anomalous diffusion behavior of the reaction coordinate $s$ for various cases in 3D [see Fig. 5(b)]. We compare unforced translocation to a pore escape, the model of which we implemented by placing a polymer halfway in an infinitely long pore [see Fig. 1(c)]. Hereby, the entropic contributions on the cis side from dimensions perpendicular to the pore axis are eliminated. The polymer was let relax to equilibrium configuration while keeping the middle bead at the exit of the pore stationary. For both the unforced translocation and the pore escape model, the meansquared displacement of the reaction coordinate $s$ scales with time, i.e., $\left\langle\delta s^{2}(t)\right\rangle \sim t^{\alpha}$, independent of the chain length $N$. In forced translocation, $\left\langle\delta s^{2}(t)\right\rangle$ does not follow a power law. These cases are discussed below. 
The entropic difference between a multidimensional heat bath and the quasi-one-dimensional pore causes the polymer to quickly escape out of the pore. We observe $N$ dependence of $\left\langle\delta s^{2}(t)\right\rangle$ at short times (not shown), which does not affect the scaling in the long-time limit. The pore escape process is seen to be clearly more subdiffusive in the long-time limit with $\alpha=0.69 \pm 0.05$ in $2 \mathrm{D}, 0.75 \pm 0.05$ in $3 \mathrm{D}(*)$, and $0.83 \pm 0.02$ in $4 \mathrm{D}$ than the regular unforced polymer translocation with $\alpha=0.81 \pm 0.02$ in $2 \mathrm{D}\left({ }^{*}\right)$ and $0.88 \pm 0.03$ in $3 \mathrm{D}(*)$, and $0.90 \pm 0.05$ in $4 \mathrm{D}$. [For results marked with $(*)$, see Fig. 5(a); others are not shown.] This was to be expected due to the additional constriction by the infinite pore. Perhaps a little less trivially, in the pore escape scaling exponents turned out to be independent of the dimension of the trans side heat bath for $2 \leq d \leq 4$. For each dimension we obtain $\beta=1.80 \pm 0.05$ for $N \in[31,51,101,251]$ (data not shown). Only the magnitude of the translocation time is diminished with increasing dimension of the trans side. Hence, based on this "simulated gedanken experiment," the dimension of the side where from, and not where to, the chain is translocating seems to determine the subdiffusive characteristics of the unforced translocation in accord with arguments giving translocation times that depend on the initial configuration, i.e., the swelling exponent of the polymer on the cis side.

In the pore escape problem the entropic force $f_{e}$ remains fairly constant throughout the process. In dimensions of 2 and 3 the free energy is roughly $F \approx-k_{b} T \ln Z_{s}$, where $Z_{s}$ is the partition sum for the polymer segment of length $s$ on the trans side, given by Eq. (1). Hence, $f_{e}=-\partial F / \partial s \sim \ln \mu+(\gamma$ $-1) / s$, where the first constant term is dominant. In dimension $d \geq 4$ we have an ideal chain and $f_{e} \simeq \ln 2 d$. Hence, $f_{e}$ is constant with respect to $s$. Denoting the entropic force as a constant $C$, in the limit of large $f_{e}$ the force balance can be written as $\xi(N-s) d s / d t=C$, where $\xi$ is the friction coefficient inside the long pore. Integrating from $t=0$ to $t=\tau$ and using $s(0)=N / 2$ and $s(\tau)=N$, we get $\tau \sim N^{2}$, which we obtained for forced translocation through a frictional pore starting from an initially straight polymer on the cis side [20]. The pore force values used there were larger than the entropic force here, so the stochastic contribution from the polymer segment inside the one-dimensional pore diminishes $\beta$ from 2 to which it will saturate when increasing the pore force. Indeed, we checked using pore force $f=1$ that the waiting times decrease linearly with the number of escaped beads, which gives $\beta=2$. In the limit of small pore friction, relevant for solvent-filled pores, $\beta$ should approach 1 since the polymer motion is only limited by the dissipation exerted on the beads escaped from the pore. Indeed, performing only microcanonical molecular dynamics inside the pore we obtain $\beta$ close to 1 in dimensions of 2 and 3.

Finally, we compare forced and unforced translocation in terms of the mean-squared displacement of the translocating polymer. In the case of forced translocation, the polymer is placed initially on the cis side, so that only the first beads are inside the pore [see Fig. 1(b)]. We have estimated that $f=1$, at which we already observed clear out-of-equilibrium characteristics, for ssDNA corresponds to a total pore force of about $1.6 \mathrm{pN}$, which is clearly smaller than experimental values [21]. The measured $\left\langle\delta s^{2}(t)\right\rangle$ for multiple force values

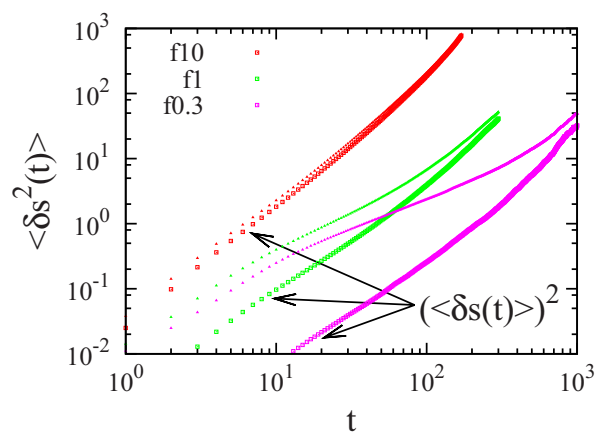

FIG. 6. (Color online) The mean-squared displacement of the reaction coordinate $\left\langle\delta s^{2}(t)\right\rangle$ is drawn in the same plot with the square of the mean displacement $\langle\delta s(t)\rangle^{2}$ for forced translocation. In the limit of high $t$ the curves are aligned. With larger force, the alignment becomes more evident already at smaller values of $t$. The data is from LD simulations in $3 \mathrm{D}$ with $N=200$ and $f=0.3,1$, and 10 (from bottom to top).

is shown in Fig. 5(b). $\left\langle\delta s^{2}(t)\right\rangle$ is seen to increase with the pore force. Unlike in the case of unforced translocation, a data collapse cannot be obtained for different $N$ [see the inset of Fig. 5(b)]. This is in qualitative agreement with [34], where "simple scaling" scenarios were ruled out using two force values. The slope is seen to increase with increasing force and in time for a constant force. For instance, for $f$ $=0.3$ the slope is initially high, then decreasing and finally increasing toward the end of translocation. This dependence on $t$ results from the change of the total friction of the chain on the cis side, $\xi_{t o t}(t) \sim s_{m}(t) \xi$. The number of "mobile beads," $s_{m}$, first increases, which decreases the slope, and then decreases, which increases the slope, as a result of all beads translocating to the trans side. See Ref. [21] for details. At high force values crowding of the beads on the trans side also has a contribution.

From Fig. 6, it is evident that $\left\langle\delta s^{2}(t)\right\rangle \neq\langle\delta s(t)\rangle^{2}$ at small $f$, but that $\left\langle\delta s^{2}(t)\right\rangle \rightarrow\langle\delta s(t)\rangle^{2}$ as $f$ is increased. This is another indication of translocation becoming more deterministic with increasing $f$, as it should due to the dynamics far from thermal equilibrium being determined solely by a simple balance between the frictional and the driving pore forces.

For forced translocation, our measured $\left\langle\delta s^{2}(t)\right\rangle$ distinctively differs from the power law obtained using another Langevin model [17]. Some of the authors therein have published results reporting two slopes with different exponents, instead [34]. Their observation of the chain elongation on the cis side is in accord with our previous results and our argument based on the simple force balance [20,21], similar in nature to the one proposed by Sakaue [22].

Also, it is evident from the results presented here and in $[20,21]$ that the pore plays a central role in polymer translocation. Our pore model is a homogeneous cylinder whereas others $[17,35]$ have used a wall of immobile (pointlike) particles through which the pore is implemented by removing a single particle. The latter implementation of the pore does not result in a homogeneous pore potential. The properties of the pore clearly have substantial contribution in the forced but not in the unforced translocation, a natural consequence of the forced translocation being a highly out-of-equilibrium 
process in the biologically and experimentally relevant force regime.

\section{SUMMARY}

We have studied unforced translocation in dimensions 2 $\leq d \leq 4$ with Langevin dynamics (LD) simulations and in 3D with stochastic rotation dynamics (SRD), including hydrodynamics with a finite Reynolds number. We have reflected the results against processes of forced translocation and a polymer escaping from an infinite pore.

First, we validated our continuous pore and wall model by studying the dependence of translocation time $\tau$ with polymer length $N$ in unforced translocation. We obtained scaling $\tau \sim N^{\beta}$ for $d \in 2,3,4$ with LD and for 3D with hydrodynamics using SRD. In dimension of 2 we obtained the scaling exponent $\beta=2.55 \pm 0.05$. In $3 \mathrm{D}$ we got $\beta=2.33 \pm 0.05$ and $\beta=2.30 \pm 0.07$ without and with hydrodynamics, respectively. Our pore design allowed us to investigate the relation between $\tau$ and $N$ in 4D, where we obtained $\beta=2.26 \pm 0.05$.

The obtained values for the scaling exponents are in agreement with the reasonably well established values obtained from simulations using wall and pore implementations based on immobile pointlike particles. These particles give rise to pore potentials curved around the pore opening. This validates our pore design and also strongly suggests that the scaling exponents for unforced translocation are universal in contrast to those for forced translocation which are highly force and model dependent. From the scaling obtained in 4D we were able to exclude a suggested lower-limit argument for $\beta$ and confirm the one presented in [9], $\beta \geq 2 \nu+1$. Hydrodynamics, although speeding up unforced translocation, was seen not to have an appreciable effect on unforced translocation time scaling with $N$, again in stark contrast with forced translocation [21]. In accordance with this, the pore being filled with solvent or not was seen not to have an effect on unforced translocation, when hydrodynamics was included.

By extracting transfer probabilities as a function of translocated beads from our unforced translocation simulations, we found out that they agreed well with those obtained from equilibrium theory $[24,25]$ in $3 \mathrm{D}$. However, the probabilities from our simulations deviated from the theoretical probabilities in 2D. This was addressed to the translocating polymer being more prone to be out of equilibrium in $2 \mathrm{D}$ than in $3 \mathrm{D}$, which we verified by measuring the relaxation and translocation times from our simulations.

Distributions of average transition times between consequent system states from our simulations proved to be closely Poissonian. We observed small deviation only at small time intervals, i.e., at large translocation velocities, where the effect of consequently translocating particles being connected to each other should most clearly be seen. It is fair to state that the anomalous diffusion behavior evident in the unforced translocation does not result from heavy-tailed waiting-time distributions as in some other cases of anomalous dynamics.

The measured mean-squared displacement of the reaction coordinate $s$, i.e., the translocated polymer beads, showed subdiffusive dynamics as expected. It was found to scale with time $t$ in the unforced translocation, $\left\langle\delta s^{2}(t)\right\rangle \sim t^{\alpha}$. The effect of hydrodynamics on this observable was more pronounced than on the scaling of translocation time with the polymer length. Here, $\left\langle\delta s^{2}(t)\right\rangle$, which was smaller when hydrodynamics was included, is a direct measure of fluctuations in the translocation process. These fluctuations are reduced through long-range correlations supported by hydrodynamics. We observed this reduction in fluctuations by hydrodynamics also through the distributions of average transition times.

To gain understanding on the translocation process we briefly investigated a simplified model where a polymer escapes from an infinite pore. We established that the scaling obtained for this process is insensitive to the dimension of the space into which the polymer is ejected, which corresponds to the trans side in the standard translocation. From this we conclude that the arguments based on the translocation time being determined by the initial configuration and the dynamics of the polymer on the cis side are indeed justified, the trans side modifying it substantially only when the polymer is driven out of equilibrium during the process, which is the case in forced translocation. Through the simple pore escape model we also showed the significance of pore friction.

Lastly, we compared forced and unforced translocations through the mean-squared displacements from the simulations. In contrast to the unforced translocation, $\left\langle\delta s^{2}(t)\right\rangle$ obtained for the forced translocation does not scale with respect to time $t$. This is another indication that the scaling exponents in forced translocation change with respect to force and the used model. Also, the square of the mean displacements, $\langle\delta s(t)\rangle^{2}$, was seen to become increasingly aligned with the mean-squared displacement as the force was increased, which is in agreement with the argued force balance governing the process at this biologically relevant force regime. This distinctively shows the more deterministic out-ofequilibrium dynamics of the forced translocation compared with the purely stochastic dynamics of the unforced translocation, shown here to take place essentially in, or very close to, thermal equilibrium.

\section{ACKNOWLEDGMENTS}

This work was supported by the Academy of Finland (Project No. 127766). The computational resources of CSC-IT Centre for Science, Finland, are acknowledged. 
[1] B. Alberts et al., Molecular Biology of the Cell (Garland Publishing, New York, 1994).

[2] A. Meller, L. Nivon, and D. Branton, Phys. Rev. Lett. 86, 3435 (2001).

[3] A. Meller and D. Branton, Electrophoresis 23, 2583 (2002).

[4] J. J. Kasianowicz, E. Brandin, D. Branton, and D. W. Deamer, Proc. Natl. Acad. Sci. U.S.A. 93, 13770 (1996).

[5] W. Storm et al., Nano Lett. 5, 1193 (2005).

[6] M. van der Laan et al., Nat. Cell Biol. 9, 1152 (2007).

[7] S. M. Simon, C. S. Peskin, and G. F. Oster, Proc. Natl. Acad. Sci. U.S.A. 89, 3770 (1992).

[8] M. Bates, M. Burns, and A. Meller, Biophys. J. 84, 2366 (2003).

[9] J. Chuang, Y. Kantor, and M. Kardar, Phys. Rev. E 65, 011802 (2001).

[10] K. Luo, T. Ala-Nissila, and S.-C. Ying, J. Chem. Phys. 124, 034714 (2006).

[11] I. Huopaniemi, K. Luo, T. Ala-Nissila, and S.-C. Ying, J. Chem. Phys. 125, 124901 (2006).

[12] J. L. A. Dubbeldam, A. Milchev, V. G. Rostiashvili, and T. A. Vilgis, Phys. Rev. E 76, 010801(R) (2007).

[13] D. Panja, G. T. Barkema, and R. C. Ball, J. Phys.: Condens. Matter 19, 432202 (2007).

[14] S. Guillouzic and G. W. Slater, Phys. Lett. A 359, 261 (2006).

[15] I. Ali and J. M. Yeomans, J. Chem. Phys. 123, 234903 (2005).

[16] M. G. Gauthier and G. W. Slater, Phys. Rev. E 79, 021802 (2009).

[17] K. Luo, S. T. T. Ollila, I. Huopaniemi, T. Ala-Nissila, P. Pomorski, M. Karttunen, S.-C. Ying, and A. Bhattacharya, Phys. Rev. E 78, 050901(R) (2008).

[18] D. Wei, W. Yang, X. Jin, and Q. Liao, J. Chem. Phys. 126,
204901 (2007).

[19] A. Milchev, K. Binder, and A. Bhattacharya, J. Chem. Phys. 121, 6042 (2004).

[20] V. V. Lehtola, R. P. Linna, and K. Kaski, Phys. Rev. E 78, 061803 (2008).

[21] V. V. Lehtola, R. P. Linna, and K. Kaski, EPL 85, 58006 (2009).

[22] T. Sakaue, Phys. Rev. E 76, 021803 (2007).

[23] M. Fyta, S. Melchionna, S. Succi, and E. Kaxiras, Phys. Rev. E 78, 036704 (2008).

[24] W. Sung and P. J. Park, Phys. Rev. Lett. 77, 783 (1996).

[25] M. Muthukumar, J. Chem. Phys. 111, 10371 (1999).

[26] E. Eisenriegler, Polymers Near Surfaces (World Scientific, Singapore, 1993).

[27] W. van Gunsteren and H. Berendsen, Mol. Phys. 34, 1311 (1977).

[28] A. Malevanets and R. Kapral, J. Chem. Phys. 110, 8605 (1999)

[29] J. Cardy and S. Redner, J. Phys. A 17, L933 (1984).

[30] A. R. Conway and A. J. Guttmann, Phys. Rev. Lett. 77, 5284 (1996).

[31] http://www.ms.unimelb.edu.au/iwan/saw/SAW_ser.html

[32] N. Madras and G. Slade, The Self-Avoiding Walk (Birkhuser, Boston, 1996).

[33] M. Doi and S. F. Edwards, The Theory of Polymer Dynamics (Oxford Science Publications, Oxford, 1986).

[34] A. Bhattacharya, W. H. Morrison, K. Luo, T. Ala-Nissila, S.-C. Ying, A. Milchev, and K. Binder, Eur. Phys. J. E 10, 1140 (2009).

[35] M. G. Gauthier and G. W. Slater, Eur. Phys. J. E 25, 17 (2008). 